\title{
Incidência de lesões em motociclistas praticantes de trilhas*
}

\section{Incidence of Injuries in Motorcyclists Practitioners from Trails}

\author{
Davi Leal Sousa ${ }^{1}$ Kelson Nonato Gomes da Silva ${ }^{1}$ Eduardo Ferreira ${ }^{1}$ Flávio Rogério de Sousa Morais ${ }^{1}$ \\ ${ }^{1}$ Centro de Ciências da Saúde, Universidade Estadual do Piauí (UESPI), \\ Teresina, PI, Brasil \\ Rev Bras Ortop 2020;55(6):728-735. \\ Endereço para correspondência Davi Leal Sousa, Centro de Ciências \\ da Saúde, Universidade Estadual do Piauí, Rua Olavo Bilac, 2335, \\ Centro (Sul), Teresina, PI, 64001-280, Brasil, \\ (e-mail: davi_ipiranga@hotmail.com).
}

\section{Resumo \\ Palavras-chave \\ - esportes \\ - veículos off-road \\ - lesões esportivas \\ - motocicletas}

Objetivo Investigar a incidência, os mecanismos, os tipos de lesão, as regiões anatômicas mais acometidas, e os fatores que podem levar a lesões nos motociclistas praticantes de trilhas.

Métodos Trata-se de uma pesquisa observacional do tipo retrospectivo, na qual foi realizada análise com 47 motociclistas praticantes de trilhas. Os dados foram coletados através da aplicação de um inquérito de morbidade referida (IMR), que incluiu informações sobre lesões e seus mecanismos.

Resultados Ao analisar a amostra, verificou-se que os tipos de lesões com maior incidência foram abrasão e contusão. As regiões anatômicas mais acometidas foram o ombro e o joelho, e o mecanismo de lesão mais comum foi a derrapagem ou perda da tração.

Conclusão Os trilheiros estão expostos a fatores de risco e, consequentemente, às quedas, sendo importante desenvolver mais equipamentos de proteção, em especial para o ombro e para o joelho.

Objective To investigate the incidence, mechanisms, types of injury, most affected anatomical regions, and the factors leading to injuries in trail bikers.

Methods This was an observational retrospective research, analyzing 47 trail bikers. Data were collected through application of a referenced morbidity survey (MRI), which included information on injuries and their mechanisms.

Results The with the highest incidence were abrasion and bruise, and the most affected anatomical regions were the shoulder and knee. And the most common injury mechanism was skidding or loss of traction.

Conclusion Trail bikers are exposed to risk factors and, consequently, to falls, and it is important to develop more protective equipment, especially for the shoulder and knees.

Trabalho feito no Centro de Ciências da Saúde, Universidade Estadual do Piauí (UESPI), Teresina, PI, Brasil.

recebido

07 de Maio de 2019

aceito

05 de Dezembro de 2019
DOI https://doi.org/

10.1055/s-0040-1702949. ISSN 0102-3616.
Copyright $\odot 2020$ by Sociedade Brasileira License terms de Ortopedia e Traumatologia. Published by Thieme Revinter Publicações Ltda, Rio de Janeiro, Brazil 


\section{Introdução}

A prática do motociclismo off-road surgiu no Brasil na década de 70, quando entusiastas por motos de todo país, colocavam suas motocicletas em trilhas como forma alternativa de diversão dos fins de semana. ${ }^{1}$

No contexto atual, as práticas do motociclismo off-road estão cada vez mais diversificadas e difundidas no território brasileiro, além de incluir modalidades competitivas (como rally, enduro, trial, e motocross), existem as trilhas na natureza, que não têm caráter competitivo. Trilhas com motos são utilizadas como esporte pelos seus praticantes e têm como objetivo superar as dificuldades de acesso e transpor obstáculos impostos pela natureza, como, por exemplo, pedras; erosões; lama; e descidas e subidas ingremes. ${ }^{2}$

Desde a sua criação, no início dos anos 20, o motociclismo off-road vem ganhando popularidade, com milhões de participantes em todo o mundo. ${ }^{3,4}$ Essa modalidade esportiva proporciona vários fatores positivos aos seus praticantes, tais como alcançar mudanças benéficas na saúde e aptidão física que contribuem para melhorar a qualidade de vida, gerando, portanto, menos fatores de risco de doenças futuras. ${ }^{5-7}$ Por outro lado, com o crescimento do número de pessoas que aderem à prática de trilhas com moto, elevam-se também a taxa de risco e a incidência de lesões. ${ }^{3,8}$

A frequência de lesão em praticantes de trilhas com motocicletas é bastante elevada. ${ }^{8,9}$ Entorses, ${ }^{10,11}$ fraturas, ${ }^{12-15}$ luxações, ${ }^{12,15}$ contusões, ${ }^{16}$ lesões ligamentares, ${ }^{12-15}$ e ferimentos ${ }^{14,15}$ têm sido descritas na literatura, como as lesões mais comuns causadas por esse esporte. Nesse sentido, o desenvolvimento de estratégias para prevenção das lesões deve conter investigação de potenciais fatores de riscos, tais como: aptidão física e mental, equipamentos de proteção, leis específicas para motos off-road, e mecanismos de lesão. ${ }^{13,14,17,18}$ Conhecer bem esses fatores é de fundamental importância, tanto para quem for responsável pela reabilitação do indivíduo lesionado, como para quem é responsável pelo treinamento do mesmo, quando se trata de um atleta. ${ }^{19}$

Lesões em modalidades off-road, especialmente em trilhas com motocicletas, ainda precisam de pesquisas relacionadas, devido ao fato de serem poucas as referências encontradas na literatura abordando especificamente essa modalidade. Nesse sentido, o objetivo do presente estudo foi investigar a incidência, mecanismos, tipos de lesão, regiões anatômicas mais acometidas, e os fatores que podem levar a lesões nos motociclistas praticantes de trilhas.

\section{Metodologia}

Trata-se de uma pesquisa observacional do tipo retrospectivo, buscando informações sobre lesões referidas por motociclistas praticantes de trilhas (trilheiros). $O$ presente estudo foi realizado na $2^{\mathrm{a}}$ edição do trilhão da juventude, em julho de 2018, na cidade de Ipiranga, no Piauí. Todos os participantes foram informados e convidados a participar da pesquisa (70). No entanto, 20 destes não estavam presentes ou não responderam ao inquérito, sendo incluídos 50 no estudo.
A presente pesquisa foi apreciada e aprovada pelo Comitê de Ética em Pesquisa sob o parecer de $n^{\circ} 2.932 .053$.

Os critérios de inclusão foram motociclistas do off-road de ambos os sexos que praticam a modalidade de trilhas em qualquer faixa etária, devidamente inscritos no $2^{\circ}$ trilhão de juventude. Dos 50 participantes que aceitaram participar do estudo, 3 não responderam ao questionário de forma adequada, restando assim 47 trilheiros. Todos os indivíduos da presente pesquisa foram informados dos objetivos da pesquisa e estudados, respeitando as normas de pesquisa envolvendo seres humanos (Res. CNS 466/2012) do Conselho Nacional de Saúde. Também foi solicitada a autorização para utilização das informações fornecidas.

A coleta de dados para a execução do trabalho foi conduzida antes do evento e obtida por meio de inquérito de morbidade referida (IMR), que é um procedimento adequado para investigações sobre o estado de saúde de grupos populacionais específicos. ${ }^{20} \mathrm{O}$ questionário (anexo 1) foi elaborado seguindo o modelo descrito por Gabriela ${ }^{19} \mathrm{e}$ modificado segundo a realidade da modalidade abordada nessa pesquisa, solicitando informações sobre o tipo de lesão, região anatômica afetada, mecanismo de lesão, fatores que podem ter contribuído para ocasionar a lesão, tempo de prática do esporte, além de informações pessoais (sexo, idade, altura, e peso). Todo o processo de aplicação dos questionários foi conduzido pelo pesquisador juntamente com dois auxiliares que foram devidamente treinados para esse estudo. Foi considerada lesão desportiva qualquer afecção musculoesquelética resultante da prática de trilha com motocicletas.

Após a coleta dos dados através do IMR, as informações foram digitadas e tabuladas em um banco de dados específicos para análise estatística dos mesmos. O banco de dados, bem como as tabelas e gráficos, foram construídos no Microsoft Excel 2010 (Microsoft Corp., Redmond, WA, EUA). Para a análise dos dados, foi utilizado o software $R$ version 3.4.3 (The R Foundation for Statistical Computing, Viena, Áustria), e o teste do Qui-quadrado foi adotado para a análise da significância estatística dos resultados obtidos, sendo considerado o nível alfa de significância 0,05 (5\%).

\section{Resultados}

Participaram desse estudo 47 trilheiros com idade entre 18 a 50 anos, todos do sexo masculino. Desses, 20 relataram nunca ter sofrido qualquer tipo de lesão, e 27 afirmaram já ter sofrido uma ou mais de uma lesão durante a prática de trilhas não competitivas.

A - Tabela 1 mostra as características dos trilheiros com lesão e sem lesão $(n=47)$ que participaram do estudo. A média de idade foi de 24,95 anos, altura média de $1,76 \mathrm{~m}$, peso médio de $76,55 \mathrm{Kg}$, índice de massa corpórea (IMC) médio de 24,18 , indicando peso adequado, e o tempo médio de prática do esporte foi de 2,49 anos para o grupo que não sofreu lesão. Já para o grupo que sofreu lesão, a média de idade foi de 32,11 anos, uma altura média de $1,73 \mathrm{~m}$, peso médio de $83,20 \mathrm{Kg}$, IMC médio de 27,84, indicando sobrepeso, e o tempo médio de prática do esporte foi de 6,14 anos. 
Tabela 1 Perfil sociodemográfico dos trilheiros $(n=47)$ participantes da pesquisa

\begin{tabular}{|c|c|c|c|c|c|}
\hline \multirow[t]{2}{*}{ Variáveis } & \multicolumn{2}{|l|}{ Com lesão } & \multicolumn{2}{|l|}{ Sem lesão } & \multirow[t]{2}{*}{ Valor de $p$} \\
\hline & $\begin{array}{l}\text { Frequência } \\
\text { absoluta }\end{array}$ & $\begin{array}{l}\text { Frequência } \\
\text { relativa (\%) }\end{array}$ & $\begin{array}{l}\text { Frequência } \\
\text { absoluta }\end{array}$ & $\begin{array}{l}\text { Frequência } \\
\text { relativa (\%) }\end{array}$ & \\
\hline \multicolumn{6}{|l|}{ Faixa etária } \\
\hline $18-30$ & 11 & $39,29 \%$ & 17 & $60,71 \%$ & \multirow[t]{2}{*}{$<0,05^{* *}$} \\
\hline $31-50$ & 16 & $84,21 \%$ & 3 & $15,79 \%$ & \\
\hline \multicolumn{6}{|l|}{ Altura } \\
\hline $1,62-1,75$ & 18 & $66,67 \%$ & 9 & $33,33 \%$ & \multirow[t]{2}{*}{$>0,05$} \\
\hline $1,76-1,89$ & 9 & $45,00 \%$ & 11 & $55,00 \%$ & \\
\hline \multicolumn{6}{|l|}{ Peso } \\
\hline $60-80$ & 13 & $54,17 \%$ & 11 & $45,83 \%$ & \multirow[t]{2}{*}{$>0,05$} \\
\hline $81-109$ & 14 & $60,87 \%$ & 9 & $39,13 \%$ & \\
\hline \multicolumn{6}{|c|}{ Índice de massa corpórea } \\
\hline Peso adequado & 11 & $45,83 \%$ & 13 & $54,17 \%$ & \multirow[t]{4}{*}{$>0,05$} \\
\hline Sobrepeso & 9 & $69,23 \%$ & 4 & $30,77 \%$ & \\
\hline Obesidade & 7 & $77,77 \%$ & 2 & $22,23 \%$ & \\
\hline Baixo peso & 0 & $0,00 \%$ & 1 & $100,00 \%$ & \\
\hline \multicolumn{6}{|c|}{ Tempo que pratica trilha } \\
\hline 2 Meses-5 anos & 13 & $40,63 \%$ & 19 & $59,37 \%$ & \multirow[t]{2}{*}{$>0,05$} \\
\hline 6 Anos-25 anos & 14 & $93,33 \%$ & 1 & $6,67 \%$ & \\
\hline
\end{tabular}

*Teste Qui-quadrado (95\% de confiança). ${ }^{* *}$ Significância estatística $(\alpha=0,05)$.

Foram identificadas 56 lesões, pois houve trilheiros que se lesionaram mais de uma vez. Os tipos de lesões com maior incidência foram a abrasão e a contusão, ambas com 23,21\% (-Figura 1). Quanto à região anatômica mais acometida, o ombro apresentou o maior índice, com 23,53\%, seguido do joelho, com 13,73\% (-Figura 2).
Outras lesões também foram mencionadas no presente estudo, sendo elas: cãibras na panturrilha e na coxa, fraturas de clavícula e costelas, e uma amputação traumática do polegar.

A lesão no ombro foi a mais frequente nos membros superiores, afetando $38,71 \%$ dos indivíduos da amostra, sendo a abrasão (12,90\%), contusão (9,68\%), fratura

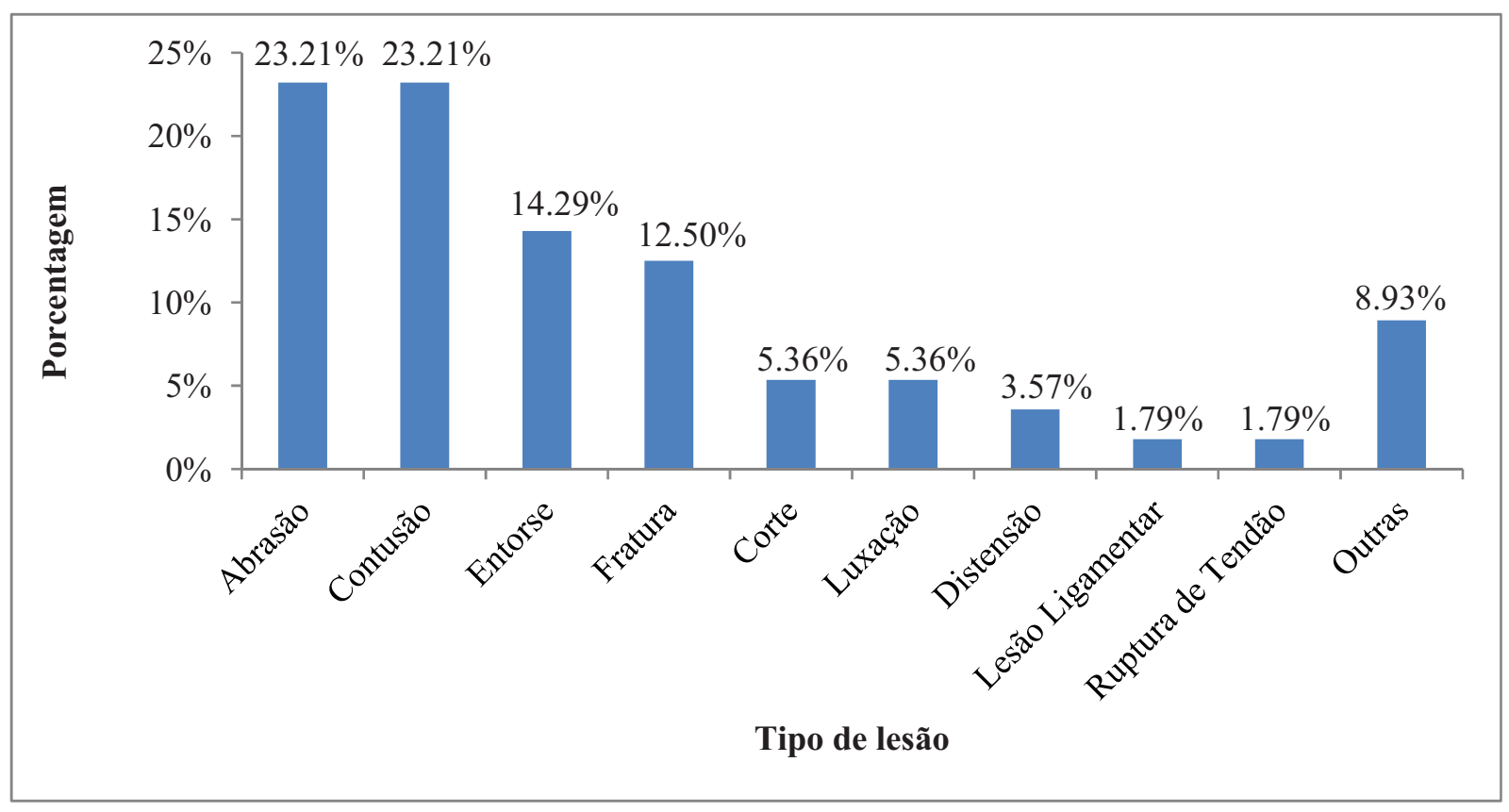

Fig. 1 Distribuição dos tipos de lesões relatadas pelos trilheiros. 


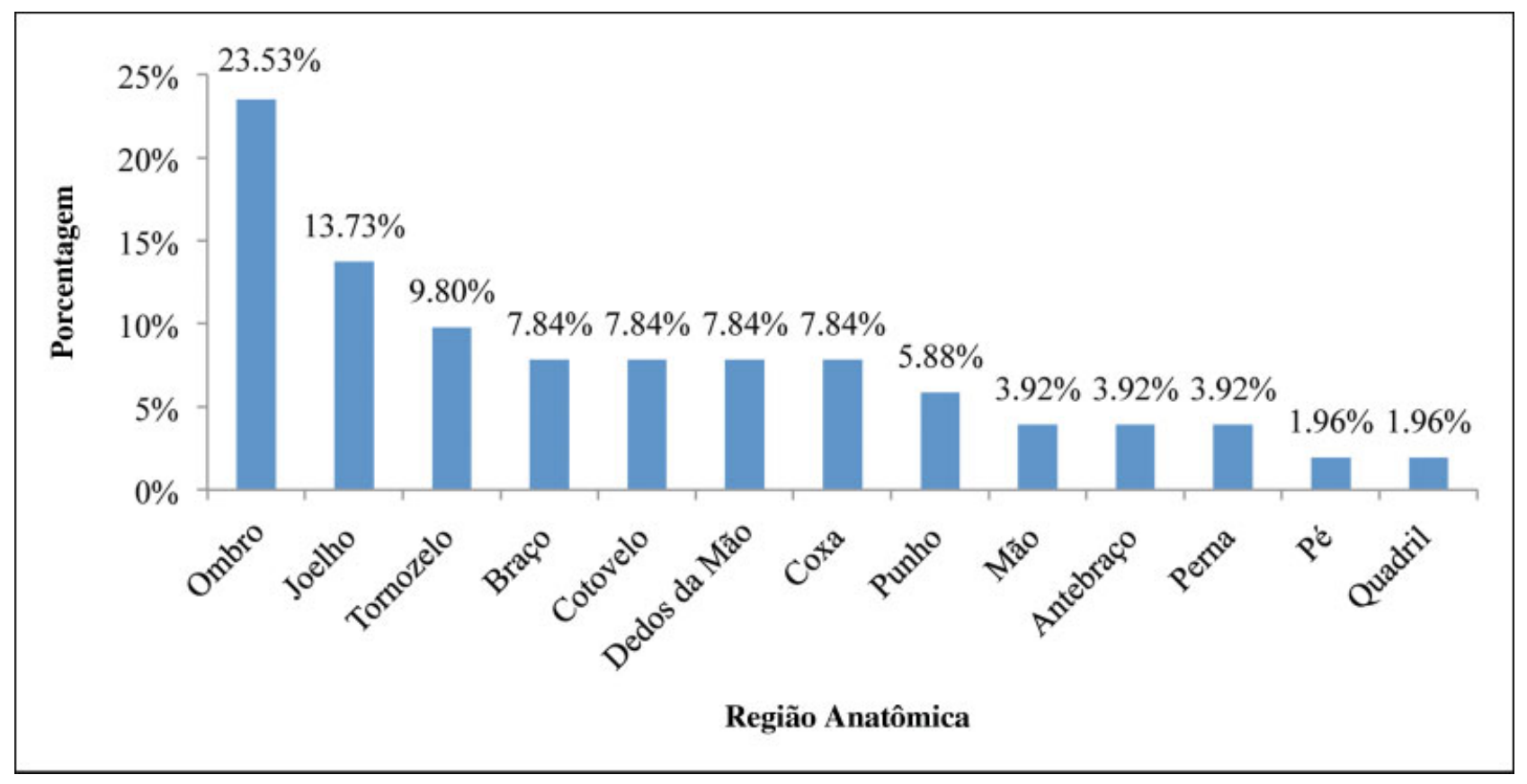

Fig. 2 Distribuição da região anatômica lesionada relatada pelos trilheiros.

(9,68\%), e luxação (6,45\%), respectivamente, os tipos de lesões mais frequentes no ombro. Logo em seguida, aparecem as lesões do braço, cotovelo, e dedos das mãos, todas com incidência de $12,90 \%$, sendo que o tipo de lesão mais comum no braço foi abrasão $(6,45 \%)$, seguida de contusão e cortes (3,23\%); no cotovelo, a abrasão e contusão (6,45\%) foram os tipos de lesão mais relatada; e nos dedos da mão, ruptura de tendão, luxação, entorse, e cortes aparecem com $3,23 \%$ cada uma.

O joelho foi a região mais lesionada nos membros inferiores (35\%), sendo o tipo de lesão mais comum foi a contusão (15\%), seguida de abrasão (10\%), e fratura e lesão ligamentar, ambas com $5 \%$. O tornozelo aparece logo em seguida, correspondendo

Tabela 2 Distribuição dos mecanismos de lesão relatados pelos trilheiros participantes da pesquisa

\begin{tabular}{|l|l|l|l|l|}
\hline $\begin{array}{l}\text { Mecanismos } \\
\text { de lesão }\end{array}$ & \multicolumn{2}{|l|}{$\begin{array}{l}\text { Frequência } \\
\text { absoluta }\end{array}$} & \multicolumn{2}{l|}{$\begin{array}{l}\text { Frequência } \\
\text { relativa (\%) }\end{array}$} \\
\hline $\begin{array}{l}\text { Derrapagem ou } \\
\text { perda da tração }\end{array}$ & 11 & & 35,48 & \\
\hline Curva & & 8 & & 25,81 \\
\hline Reta & & 3 & & 9,68 \\
\hline $\begin{array}{l}\text { Colisão com um } \\
\text { objeto imóvel }\end{array}$ & 10 & & 32,26 & \\
\hline Pedra & & 7 & & 22,58 \\
\hline Árvore & & 3 & & 9,68 \\
\hline $\begin{array}{l}\text { Colisão com um } \\
\text { objeto móvel }\end{array}$ & 4 & & 12,90 & \\
\hline Moto & & 4 & & 12,90 \\
\hline Capotamento & 4 & 4 & 12,90 & 12,90 \\
\hline Após um salto & 2 & 3 & 6,45 & 6,45 \\
\hline Total & 31 & 31 & 100 & 100 \\
\hline
\end{tabular}

a $25 \%$ das lesões, sendo que as entorses predominaram (25\%). A coxa aparece com $20 \%$, a distensão (10\%), a abrasão (5\%), e a contusão (5\%) foram os tipos de lesão mais comuns da coxa.

$\mathrm{Na}$ - Tabela 2, verificam-se os mecanismos de lesão. A derrapagem, ou perda de tração, foi a principal causa dos acidentes (35,48\%), sendo que $25,81 \%$ dos trilheiros relataram que caíram e sofreram a lesão em uma curva, enquanto que $9,68 \%$ sofreram a lesão em uma reta. Outros 32,26\% sofreram lesão após colisão com um objeto imóvel, sendo que as colisões com pedras (22,58\%), seguidas de colisões com árvores $(9,68 \%)$ foram as mais comuns.

Quanto à direção da queda, 40,74\% dos trilheiros caíram para o lado esquerdo, $33,33 \%$ relataram que a queda aconteceu para o lado direito, 22,22\% relataram que a queda foi sobre o guidão, e 3,70\% afirmaram ter sofrido a lesão sem ocorrência de queda (-Figura 3).

Quando analisados os fatores de riscos contribuintes para ocasionar as lesões, $25,81 \%$ dos trilheiros relataram que a alta velocidade foi o que contribuiu para causar a lesão. Em 12,90\%, a causa foi a falta de equipamento de proteção; a ingestão de bebidas alcoólicas e as imprudências, ambas, representaram 9,68\% dos fatores de risco (-Figura 4 ).

\section{Discussão}

De acordo com os achados da presente pesquisa, constatouse que a idade média dos trilheiros que sofreram lesões é de 32,11 anos, o peso $83,20 \mathrm{~kg}$, a altura $1,73 \mathrm{~m}$, o IMC de 27,84, e o tempo médio de prática do esporte foi de 6,14 anos. Essas variáveis do perfil sociodemográfico fornecem informações relevantes quanto ao risco de ser acometido por lesões. Mary et al. ${ }^{10}$ realizaram um estudo com motociclistas off-road em Victoria, Austrália, verificaram que jovens inexperientes no esporte, de menor estatura e baixo peso podem estar mais propensos a lesão. 


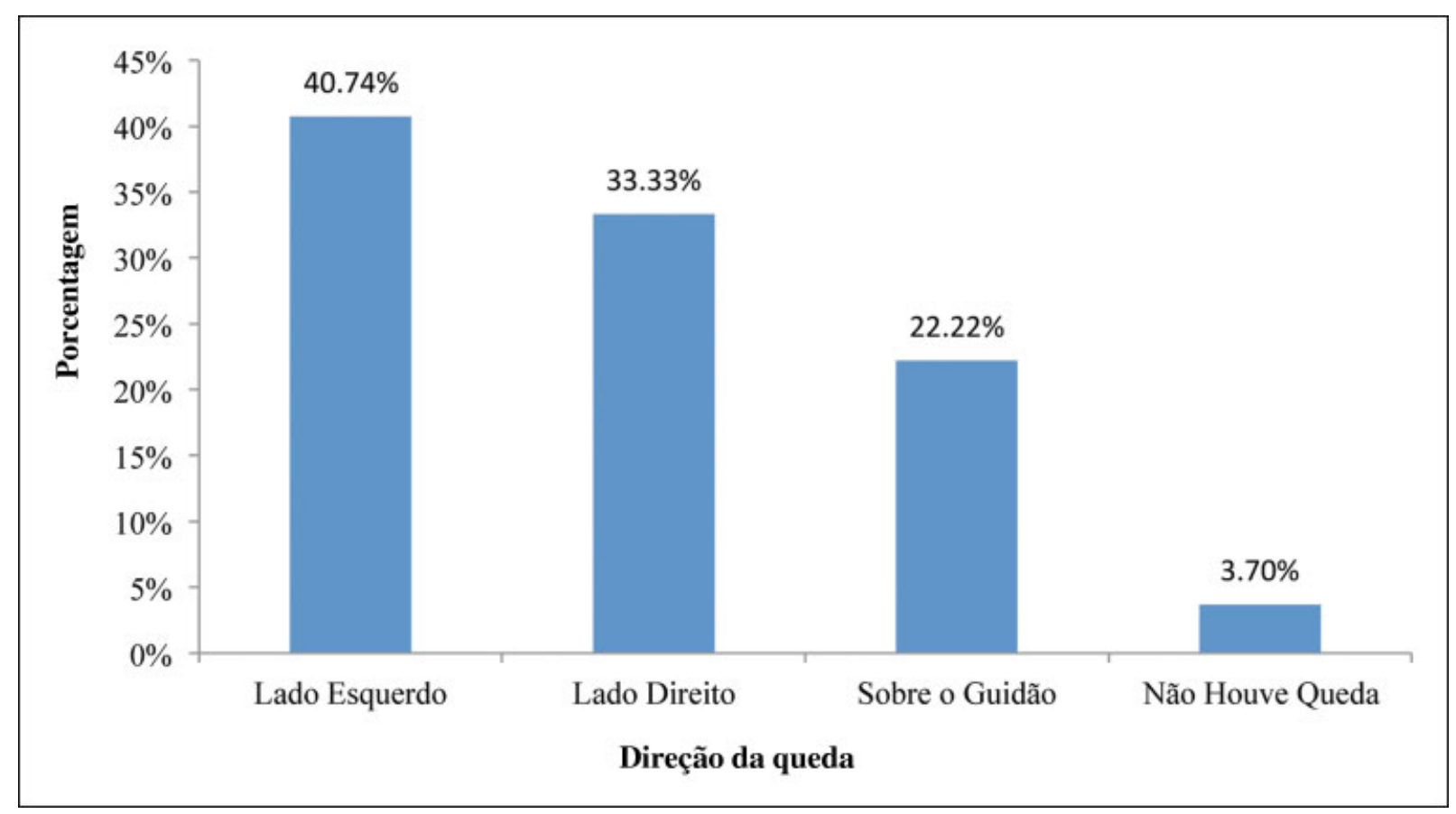

Fig. 3 Direção para qual acorreu a queda com o motociclista de trilha.

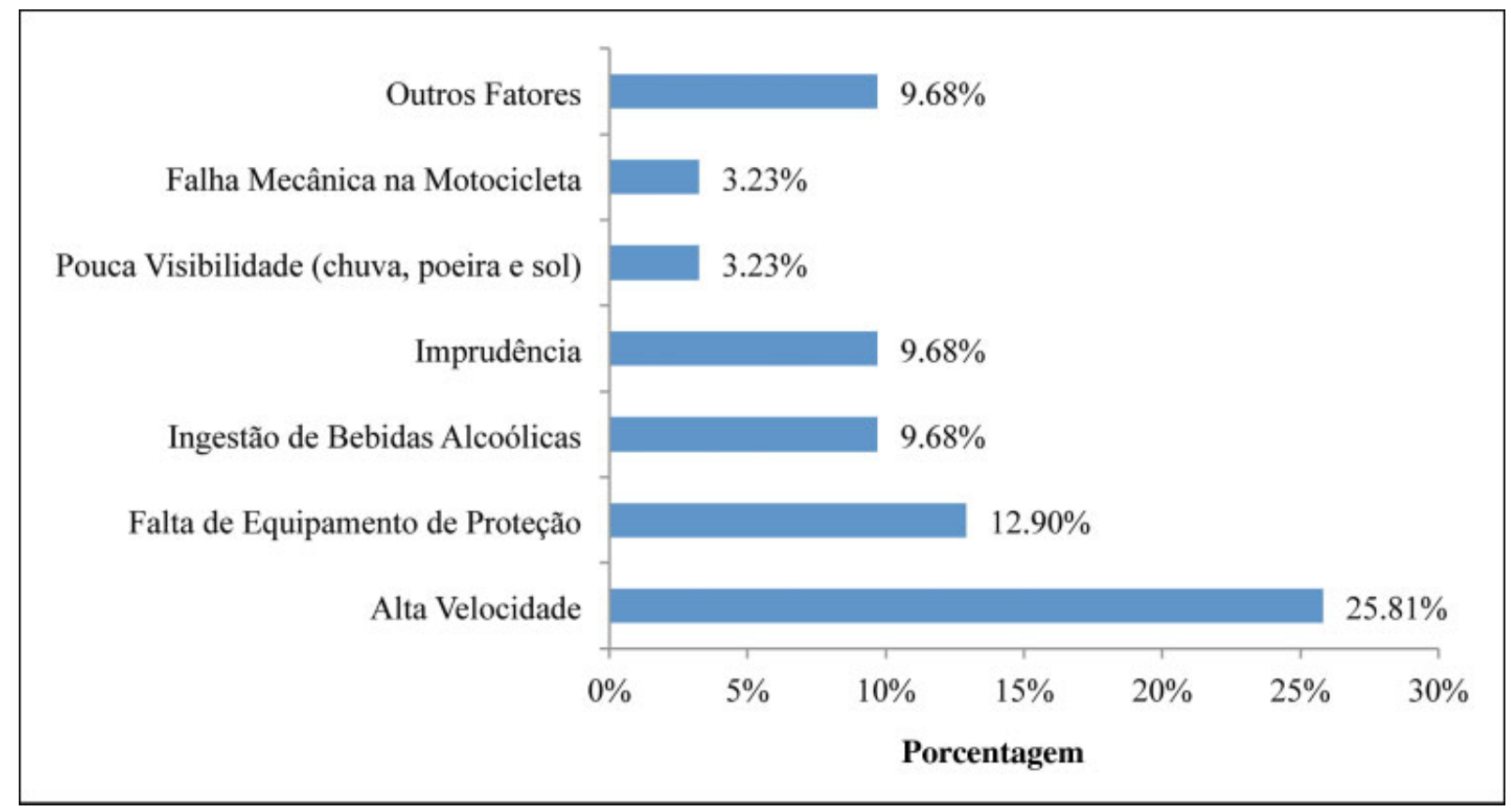

Fig. 4 Fatores de riscos relatados que contribuíram para acontecer a lesão.

No estudo de Tomida et al. ${ }^{21}$ com motociclistas de elite no Japão, investigaram se a idade e experiência dos corredores de motocross e trial e, ao correlacionar estas variáveis com lesões, concluíram que não há relação entre a idade e experiência com o surgimento de lesões. Esta afirmação condiz parcialmente com o resultado da presente pesquisa, em que o resultado do teste do Qui-quadrado mostra que a idade foi à única variável estatisticamente significativa, ou seja, os atletas reagem às lesões de forma diferente dependendo da faixa etária.

Jason et al. ${ }^{22}$ realizaram uma análise longitudinal com jovens abaixo de 20 anos, que receberam atendimento médico de emergência em Ontário, Canadá, devido a um acidente de veículo off-road entre $1^{\circ}$ de abril de 2002 e 31 de março de 2014. Os dados do estudo constataram que jovens com menos de 20 anos estão associados a um aumento de cuidados médicos de emergência, por causa de acidentes com veículos off-road. Os pesquisadores ainda ressaltam que as lesões são parcialmente causadas nessa população por supervisão inadequada e comportamento impulsivo. Em nosso estudo, foi constatado que a idade está relacionada à lesão, mais não foi possível analisar se os jovens com menos de 20 anos estão mais suscetíveis por conta da pequena participação de jovens no estudo. 
A média do IMC $(27,84)$ dos trilheiros do presente estudo aponta um sobrepeso, o que é corroborado pelo estudo de Gobbi et al. ${ }^{17}$ Esse trabalho analisou as características fisiológicas de pilotos do motocross, rally, e enduro, e identificou que pilotos de rally propendiam a ter excesso de peso. Na revisão de literatura feita por Khanna et al., ${ }^{23}$ foi apontado que pilotos de enduro estão no limite do peso normal. Apesar do teste do Quiquadrado não ter apresentado significância estatística sobre o peso nesse estudo, algumas pesquisas apontam que estar acima do peso em esportes off-road é um fator agravante de lesões, pois torna mais difícil a pilotagem, especialmente ao subir ou descer morros e atravessar lama. ${ }^{17,23}$

No presente trabalho foi observado que a maioria das lesões ocorreram nos membros superiores, o que condiz com os achados de Day et al. ${ }^{11}$ que, em 2016, realizou um estudo com crianças de 0 a 17 anos que sofreram ferimentos enquanto estavam pilotando uma motocicleta off-road para fins recreativos. Outro estudo que constatou esse mesmo resultado foi o de Benjamin et al., ${ }^{12}$ realizado em 2015, que mostrou que as fraturas da extremidade superior foram as mais prevalentes nos motociclistas que participaram do rally Dakar em 2013 e 2014.

No estudo realizado por Sabeti-Aschraf et al. ${ }^{13}$ com motociclistas que participaram do Erzberg Rodeo entre 2005 e 2007, uma corrida de enduro que acontece na Áustria, os autores identificaram que as lesões mais frequentes foram a contusões e abrasões. Sandler et al. ${ }^{24}$ também constataram que as contusões e abrasões foram as lesões mais comuns em crianças feridas ao pilotar veículos off-road. Esse tipo de lesão geralmente ocorre quando há um deslize do pneu dianteiro provocando a queda do piloto. ${ }^{13} \mathrm{Já}$ em um trabalho realizado

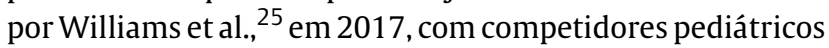
de motocross, os tipos de lesões mais encontrados foram fraturas. No estudo de Padegimas et al., ${ }^{26}$ em 2016, as entorses ocorreram com mais frequência. Esses achados se assemelham aos nossos, visto que as entorses e as fraturas foram o terceiro e o quarto tipo de lesão mais incidente.

Mary et al. ${ }^{10}$ identificaram em seu estudo, que as regiões anatômicas mais lesionadas em motociclistas do off-road foram: ombro, joelho, punho e tornozelo, o que se assemelha aos dados encontrados no presente artigo, em que as regiões mais acometidas foram: ombro, joelho, tornozelo, braços, cotovelo, dedos da mão, e coxa. Em uma revisão sistemática realizada por Khanna et al. ${ }^{23}$ em 2015, os autores identificaram que as mãos, braços, e ombros foram os pontos de impacto mais comuns após queda dos motociclistas de enduro. Os autores ressaltam ainda que o esforço gerado pelos membros inferiores dos motociclistas é maior que o gerado pelos membros superiores; este fato pode ser o motivo das lesões em membros inferiores.

Outras lesões foram relatadas pelos participantes desse estudo, sendo elas cãibras na panturrilha e coxa, fraturas de clavícula e costelas, e amputação traumática do polegar. A literatura também relata a ocorrência dessas lesões, como nos estudos de Winkes et al. ${ }^{27}$ e Sabeti-Aschraf et al., ${ }^{28}$ que relatam cãibras em antebraços de pilotos de motocross e enduro. As fraturas de clavícula e costelas foram relatadas nos estudos de Nona e Richard, ${ }^{15}$ que analisaram durante 4 anos pilotos de enduro, durante o 6-Day Enduro em quatro países Austrália, Holanda, Estados Unidos, e Polônia, e no de Benjamin et al. ${ }^{12}$ com pilotos de rally. As amputações foram relatadas por Mary et al. ${ }^{10}$ e Sawyer et al. ${ }^{29}$ Os autores ainda afirmam que as amputações ocorrem quando o membro fica preso na corrente de transmissão, e que é uma característica de lesão associada à quadriciclos.

Segundo Sabeti-Aschraf et al., ${ }^{13}$ a perda de tração (derrapagem) foi o mecanismo de lesão que ocorreu com maior frequência, confirmando assim os achados desse estudo. Já no estudo de Nona e Richard, ${ }^{15}$ os mecanismos de lesão que ocorreram com maior frequência foram: colisão com um objeto imóvel, perda de tração (derrapagem) e colisão com objeto móvel, respectivamente. No estudo de Schönle e Fachklinik, ${ }^{30}$ conduzido durante o campeonato mundial de enduro com pilotos de 24 nações, foi apontado que 7,1\% das lesões aconteceram por causa de capotamento. O mecanismo de queda após um salto é mais comum na modalidade de motocross. ${ }^{18}$ Esses resultados se assemelham com os achados do presente estudo.

Em um trabalho realizado por Larson e McIntosh ${ }^{31}$ envolvendo motociclistas do off-road nos Estados Unidos, o lado para qual os pilotos caíram com maior frequência foi o esquerdo. No presente trabalho, também foram apontadas as quedas do lado esquerdo como sendo as mais comuns. Esses mesmos autores verificaram em seu trabalho que as quedas para o lado esquerdo são mais frequentes, talvez devido à localização do pedal de marcha, operados pelo lado esquerdo da motocicleta. Outros estudos também apontam a direção da queda dos pilotos, como o de Khanna et al., ${ }^{23}$ que identificou que as quedas sobre 0 guidão foram as mais comuns, seguidas de quedas do lado direito e esquerdo. Esses dados se assemelham aos dados do presente trabalho, visto que esses foram os três lados que ocorreram mais frequentemente nos casos dos trilheiros.

Vários estudos têm apontado diversos fatores de riscos como contribuintes para ocasionar lesões, tais como: alta velocidade, ${ }^{3,13}$ falta de equipamento de proteção, ${ }^{10}$ ingestão de bebidas alcoólicas, ${ }^{9,10}$ imprudências, ${ }^{22}$ pouca visibilidade, $^{13,15}$ e falhas mecânicas. ${ }^{13,32}$ No estudo de Roxanne et al., ${ }^{8}$ que investigou lesões em pilotos americanos de motocross e motociclistas do off-road recreativo, os autores afirmam que a chance de ser ferido durante a participação em atividades com motocicleta aumenta quando o piloto está sob a influência de álcool, pilotando em altas velocidades, e não fazendo uso de equipamentos de proteção. Esses resultados foram corroborados pelo presente estudo.

\section{Conclusão}

Os trilheiros estão expostos a fatores de risco e, consequentemente, às quedas. As lesões descritas no presente trabalho ocorreram por falta de experiência, altas velocidades, e pelas próprias características do esporte, demonstrando assim a importância de se desenvolver cada vez mais equipamentos de proteção, em especial para o ombro e para os joelhos. É importante que outros estudos sejam elaborados de maneira que contribuam com mais informações para fisioterapeutas, para os demais profissionais de saúde, e para a própria população de uma maneira geral. 


\section{Conflito de Interesses}

Os autores declaram não haver conflito de interesses.

\section{Referências}

1 Off-Road. Was Brasil. Disponível em: http://wasbrasil.com.br. [Acesso em 09 maio 2018]

2 Waltrick A, Brancher EA. Jeep off-road: mais uma opção de esporte junto à natureza. JOPEF 2008;1(03):248-252

3 Sanders MS, Cates RA, Baker MD, Barber-Westin SD, Gladin WM, Levy MS. Knee injuries and the use of prophylactic knee bracing in off-road motorcycling: results of a large-scale epidemiological study. Am J Sports Med 2011;39(07):1395-1400

4 Sabeti-Aschraf M, Serek M, Pachtner T, et al. The Enduro motorcyclist's wrist and other overuse injuries in competitive Enduro motorcyclists: a prospective study. Scand J Med Sci Sports 2008; 18(05):582-590

5 Andréa SP, Giuliano GAP, Larissa ML. Atividades físicas de aventura na natureza: relações entre estilo de vida aventureiro e modo de vida rural para a melhoria da qualidade de vida. Iniciação Científica CESUMAR 2004;6(02):112-119

6 Burr JF, Jamnik VK, Shaw JA, Gledhill N. Physiological demands of off-road vehicle riding. Med Sci Sports Exerc 2010;42(07): 1345-1354

7 Burr JF, Jamnik V, Gledhill N. A cross-sectional examination of the physical fitness and selected health attributes of recreational allterrain vehicle riders and off-road motorcyclists. J Sports Sci 2010;28(13):1423-1433

8 Stiles R, Benge C, Stiles PJ, et al. Evaluation of protective equipment used among motorbike riders. Kans J Med 2018;11(02):1-13

9 Villegas CV, Bowman SM, Zogg CK, et al. The hazards of off-road motor sports: Are four wheels better than two? Injury 2016;47 (01):178-183

10 Mary OH, Erin C, Melinda C. Off-road motorcycling injury in Victoria. Hazard 2006 Melbourne: Monash University Accident Research Centre; 2006

11 Day L, Clapperton A, Berecki-Gisolf J. Off-road motorcycle injury among children aged 0-17 years in Victoria [acesso em 01 maio 2018]. Disponível em: https://www.monash.edu/muarc/research/ research-areas/home-and-community/visu/hazard

12 Benjamin D, Ahmed L, Mathieu L, et al. Musculoskeletal injuries in a resourceconstrained environment: comparing diagnostic accuracy of on-the-spot ultrasonography and conventional radiography for bone fracture screening during the Paris-Dakar rally raid. Acta Radiol Open 2015;4(05):1-7

13 Sabeti-Aschraf M, Serek M, Pachtner T, et al. Accidents and injuries in competitive Enduro motorcyclists: a prospective analysis. Knee Surg Sports Traumatol Arthrosc 2009;17(06):695-702

14 Grange JT, Bodnar JA, Corbett SW. Motocross medicine. Curr Sports Med Rep 2009;8(03):125-130

15 Colburn NT, Meyer RD. Sports injury or trauma? Injuries of the competition off-road motorcyclist. Injury 2003;34(03): 207-214
16 Gobbi A, Tuy B, Panuncialman I. The incidence of motocross injuries: a 12-year investigation. Knee Surg Sports Traumatol Arthrosc 2004;12(06):574-580

17 Gobbi AW, Francisco RA, Tuy B, Kvitne RS. Physiological characteristics of top level off-road motorcyclists. Br J Sports Med 2005; 39(12):927-931, discussion 931

18 Centers for Disease Control and Prevention (CDC). Nonfatal injuries from off-road motorcycle riding among children and teens-United States, 2001-2004. MMWR Morb Mortal Wkly Rep 2006;55(22):621-624

19 Gabriela S. Lesões agudas referidas por atletas praticantes de corrida de aventura [monografia]. Bauru: Universidade Estadual Paulista; 2006

20 Marcelo C, Edgard MJ, Liciana VAS, Aguinaldo G. Exploração de fatores de risco de lesões desportivas entre universitários de educação física: estudo a partir de estudantes de Sorocaba/SP. Rev Bras Med Esporte 2002;8(04):151-156

21 Tomida Y, Hirata H, Fukuda A, et al. Injuries in elite motorcycle racing in Japan. Br J Sports Med 2005;39(08):508-511

22 Woodfine JD, Thiruchelvam D, Redelmeier DA. Off-road vehicle crash risk during the six months after a birthday. PLoS One 2016; 11(10):e0149536

23 Khanna A, Bagouri EO, Gougoulias N, Maffulli N. Sport injuries in enduro riders: a review of literature. Muscles Ligaments Tendons J 2015;5(03):200-202

24 Sandler G, Soundappan SS, Manglick MP, et al. Pediatric "off-road vehicle" trauma: determinants of injury severity and type. Pediatr Emerg Care 2012;28(12):1328-1333

25 Williams BA, McFadden JP, Teurlings TL, Blakemore LC. Pediatric Injuries at an Annual Motocross Competition: Rates and Severity. J Pediatr Orthop 2017;37(03):e168-e173

26 Padegimas EM, Stepan JG, Stoker GE, Polites GM, Brophy RH. Epidemiology and Severity of Sports and Recreation Injuries Presenting to a Tertiary Adult Emergency Department. Phys Sportsmed 2016;44(03):263-268

27 Winkes MB, Luiten EJ, van Zoest WJ, Sala HA, Hoogeveen AR, Scheltinga MR. Long-term results of surgical decompression of chronic exertional compartment syndrome of the forearm in motocross racers. Am J Sports Med 2012;40(02):452-458

28 Sabeti-Aschraf M, Karin ZP, Serek M, Geisler M. The Effectiveness of 2 Different Therapy Modalities in the Enduro Motorcyclist's Wrist: a Prospective Randomized Sample Study. Phys Med Rehab Kuror 2010;10(04):195-200

29 Sawyer JR, Kelly DM, Kellum E, Warner WC Jr. Orthopaedic aspects of all-terrain vehicle-related injury. J Am Acad Orthop Surg 2011; 19(04):219-225

30 Schönle C, Fachklinik A. Unfallursachen und Verletzungsprophylaxe beim Enduro-Motorradsport. Sportverletz Sportschaden 1991;5(02):108-114

31 Larson AN, McIntosh AL. The epidemiology of injury in ATV and motocross sports. Med Sport Sci 2012;58:158-172

32 Chow TK, Kronisch RL. Mechanisms of injury in competitive offroad bicycling. Wilderness Environ Med 2002;13(01):27-30 


\section{Anexo 1 Lesões ocorridas durante pratica de trilhas com motocicletas}

Voluntário: 01

Sexo:___ Idade:

Tempo que pratica trilha

\section{JÁ SOFREU LESÃO PRATICANDO TRILHA NÃO COMPETI- \\ TIVA? ( ) Sim ( ) Não}

2. CORRELACIONE A PRIMEIRA COLUNA COM A SEGUNDA SOBRE AS LESÕES.

TIPOS DE LESÃO REGIÃO AFETADA

(A) CORTE (qualquer dimensão)

(B) ABRASÃO (raspagem, esfolamento, escoriações, ralada)

(C) CONTUSÃO (batida forte, impacto em uma parte do corpo)

(D) DISTENSÃO (estiramento ou rompimento das fibras musculares)

(E) LESÃO LIGAMENTAR (estiramento ou ruptura)

(F) RUPTURA DE TENDÃO (rompimento parcial ou total)

(G) LUXAÇÃO (deslocamento da posição normal de uma articulação)

(H) ENTORSE (torção brusca de uma superfície articular)

(I) FRATURA (completa ou parcial do osso) Outra

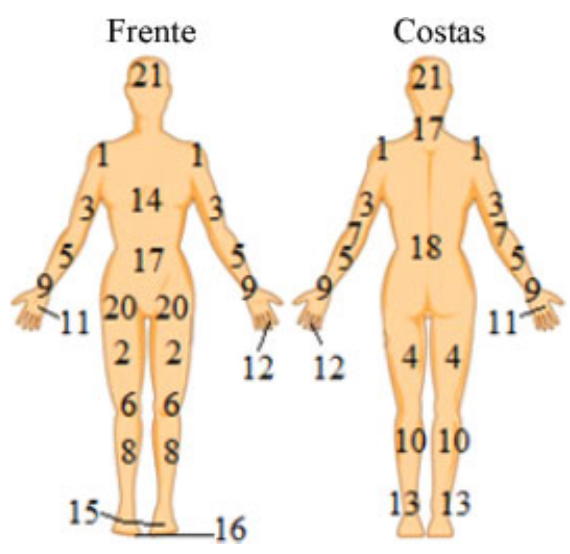

1. Ombro ( ) ( )( )

2. Coxa anterior ()()()

3. Braço ( ) ( ) ( )

4. Coxa posterior ()()()

5. Antebraço ( ) ( ) ( )
6. Joelho ( ) ( ) ( )

7. Cotovelo ()()()

8. Perna ()()()

9. Punho ()()()

10. Panturrilha ()()()

11. Mão ( ) ( ) ( )

12. Dedos da mão ( ) ( ) ( )

13. Tornozelo ( ) ( ) ( )

14. Tórax ( ) ( ) ( )

15. Pé ( ) ( )( )

16. Dedos dos pés ()()()

17. Abdome ( ) ( ) ( )

18. Coluna lombar( $)($ )( )

19. Coluna cervical( $)()($ )

20. Quadril ( ) ( ) ( )

21. Cabeça ( ) ( ) ( )

Outra

\section{MECANISMO DA LESÃO - COMO ESSA LESÃO OCORREU?}

- Colisão com um objeto imóvel: ( ) Árvore ( ) Pedra ( ) Cerca

- Colisão com um objeto móvel: ( ) Carro ( ) Animal ( ) Pedestre ( ) Moto

- Derrapagem ou perda da tração: ( ) Em uma curva ( ) Em uma reta

- Capotamento ( )

- Após um salto ( )

Outro

3.1 EM QUAL DIREÇÃO ACONTECEU A QUEDA

- Queda para frente sobre o guidão ( )

- Queda para o lado: Lado direito ( ) Lado esquerdo ( )

- Não houve queda ( )

4. FATORES QUE PODEM TER CONTRIBUÍDO PARA OCASIONARALESÃO

( ) Falta de equipamento de proteção: Qual/ Quais

( ) Falha mecânica na motocicleta

( ) Alta velocidade

( ) Ingestão de bebidas alcoólicas

( ) Falta de concentração ou atenção

( ) Falta de preparo físico

( ) Imprudência

( ) Pouca visibilidade (chuva, poeira e brilho do sol)

( ) Nenhum desses fatores

( ) Outro: 